\title{
Magnetic susceptibility from electron holes
}

\author{
R. A. Treumann ${ }^{1,{ }^{*}}$ and W. Baumjohann ${ }^{2}$ \\ ${ }^{1}$ Department of Geophysics and Environmental Sciences, Munich University, Munich, Germany \\ ${ }^{2}$ Space Research Institute, Austrian Academy of Sciences, Graz, Austria \\ * visiting: International Space Science Institute, Bern, Switzerland
}

Correspondence to: R. A. Treumann (rudolf.treumann@geophysik.uni-muenchen.de)

Received: 29 April 2013 - Accepted: 26 June 2013 - Published: 3 July 2013

\begin{abstract}
A recent theory of magnetic field amplification in electron holes is extended to derive the magnetic susceptibility of an electron-hole gas propagating in a magnetic flux tube along the ambient magnetic field. It is shown that the hole gas behaves diamagnetic adding some small amount to the well-known Landau susceptibility in the hole-carrying volume.
\end{abstract}

Keywords. Magnetospheric physics (auroral phenomena) Space plasma physics (waves and instabilities)

\section{Introduction}

In a recent paper (Treumann and Baumjohann, 2012), following earlier observations and theoretical investigations (Anderson et al., 2009; Du et al., 2011; Tao et al., 2011), we studied the magnetic effect caused by electron holes in a plasma finding the electron holes locally to amplify the magnetic field while behaving like magnetic (quasi-)particles of a certain mass, charge and magnetic moment. In the present note we consider the holes to form a quasi-particle gas. Since they exert a magnetic effect on the plasma in which they are generated and propagate, such a gas necessarily contributes to the local overall magnetic susceptibility of the plasma. This investigation adds to electron hole physics in plasmas by demonstrating one of their thermodynamic properties.

\section{Electron-hole gas}

We consider the electron-hole quasi-particle gas of density $N_{\mathrm{h}}$ to be subject to thermodynamics having adjusted to the plasma temperature $T$ when propagating inside the plasma heat bath. Actually, the holes might be colder than the sur- rounding plasma if considering the trapped electron component which belongs to the particles with energy less than the electrostatic trapping potential of the hole. However, hotter electrons travelling along the magnetic field and passing the hole without becoming trapped naturally account for thermal equilibrium conditions between the hole as an entity and the surrounding plasma.

It has been shown in Treumann and Baumjohann (2012) that each hole carries a magnetic moment $m_{\mathrm{h}}$ which is proportional to the azimuthal current $I_{\phi}$ flowing inside the hole, and the square of the hole radius $r_{\mathrm{h}}^{2}$. Both current and radius are individual properties of the hole, implying that there will be a distribution of current strengths and radii. For this reason we may consider the electron-hole gas as consisting of an entire spectrum of quasi-particles, and the magnetic moment becomes a statistical quantity

$m_{\mathrm{h}}=\frac{1}{4} r_{\mathrm{h}}^{2} I_{\phi}$

with the current $I_{\phi}$ being given by the integral

$I_{\phi}=\frac{N T \ell_{\mathrm{h}}}{B_{0}}\left\{I_{0}\left[s I_{0}\left(x_{\mathrm{h}}\right)\right]-I_{0}(s)\right\}$,

where $I_{0}(s)$ is the zero-order modified Bessel function, and $s=e U / T$ the normalized to temperature hole potential energy, $\ell_{h}$ the parallel length of the hole, and $x_{\mathrm{h}} \propto r_{\mathrm{h}}$. A more useful approximate form can be obtained by expanding the expression in braces:

$I_{\phi} \approx \frac{\ell_{\mathrm{h}} B_{0}}{4 \pi \mu_{0}} \frac{\left[I_{0} x_{\mathrm{h}}\right]^{2}-1}{1+\pi^{2} \lambda_{\mathrm{h}}^{2} / \ell_{\mathrm{h}}^{2}}\left(\frac{E_{\mathrm{h}}^{2}}{c^{2} B_{0}^{2}}\right)$.

This equation exhibits the dependence of the hole current strength $I_{\phi}$ on the ratio of the hole-electric field to external magnetic field energy. 
According to the theory of solitons and holes in a plasma. it is suggested that the dimensions of the solitons and consequently the holes (which resemble solitons in some way even though obeying a different physics) are directly related to their velocities (Buneman, 1958, 1959; Davidson, 1972; Treumann and Baumjohann, 1997; Newman et al., 2001). Since all holes have a very well defined mass, $M_{\mathrm{h}}$ (see Eq. 50 in Treumann and Baumjohann, 2012), the holes thus obey a certain distribution with respect to their momenta and kinetic energies. This distribution is not very well known, however. An approximate form has been given by Meiss and Horton (1982). Such distributions are somewhat modified Boltzmann distributions. For our purposes the modification is not of vital interest; the important point is that a gas of solitons and electron holes can approximately be described by a (onedimensional) thermal distribution of the quasi-particles.

\section{Susceptibility}

By simply assuming a thermal distribution of the holes and using the theory of magnetic moment generation developed in our previous paper, we are in the position to find the expression for the magnetic susceptibility generated in the electron-hole gas, $\chi_{\mathrm{h}}$. From fundamental thermodynamics the susceptibility is defined as the second derivative with respect to the external magnetic field $B_{0}$ of the logarithm of the grand partition function $\mathcal{Z}$ of the gas

$\chi_{\mathrm{h}}=\left.T \frac{\partial^{2}}{\partial B_{0}^{2}}\left(\frac{\log \mathcal{Z}}{V}\right)\right|_{T, V, \mu}$,

where $V$ and $\mu$ are the volume and chemical potential, respectively. We are interested only in the high temperature limit where the Bose and Fermi forms of the partition functions coincide. However, the possession of well-defined magnetic moments in Eq. (1), which in an external magnetic field imply a spectrum of energy states, qualifies electron holes for the use of the Fermi distribution, in which case we have for the grand partition function

$$
\mathcal{Z}=\prod_{s}\left[1+z \exp \left(-\beta \epsilon_{\mathrm{s}}\right)\right], \quad z \equiv \exp (\beta \mu), \beta=T^{-1},
$$

where $\epsilon_{\mathrm{s}}=p_{\|}^{2} / 2 M_{\mathrm{sh}}-m_{\mathrm{sh}} B_{0}+\hbar \omega_{\mathrm{ch}}\left(s+\frac{1}{2}\right)$ are the energy states, and $T$ is taken in energy units. Then, in the hightemperature limit $z \rightarrow 0$ is small and can be used for expanding the grand partition function. Here we included the gyration of the holes embedded in the magnetic field. Their gyration frequency is $\omega_{\mathrm{ch}}=q_{\mathrm{h}} B_{0} / M_{\mathrm{h}}$, and the corresponding energies form a discrete spectrum. When assuming that the holes have similar masses and magnetic moments, and restricting to first order in $z$ only, we then have for the logarithm $\log \mathcal{Z} \approx \frac{z L}{\pi \hbar} \sum_{s=0}^{\infty} \int_{0}^{\infty} d p_{\|} \mathrm{e}^{\left\{-\beta\left[p_{\|} / 2 M_{\mathrm{h}}-m_{\mathrm{h}} B_{0}+\hbar \omega_{\mathrm{ch}}\left(s+\frac{1}{2}\right)\right]\right\}}$

with $L$ the length along the field line. Performing the integral and sum yields an expression known from Landau diamagnetism (Landau, 1930; Landau and Lifschitz, 1965) and rewritten here for the holes

$\log \mathcal{Z} \approx \frac{z V}{\lambda_{\mathrm{hT}}^{3}}\left[1-\frac{m_{\mathrm{h}} B_{0}}{T}-\frac{1}{24}\left(\frac{\hbar \omega_{\mathrm{ch}}}{T}\right)^{2}\right]$.

This formula contains in addition the perpendicular magnetic energy of the hole, and $\lambda_{\mathrm{hT}}=\sqrt{2 \pi \hbar^{2} / M_{\mathrm{h}} T}$ is the average thermal quantum length of the holes. From statistical mechanics we know (e.g. Huang, 1987) that $z / \lambda_{\mathrm{hT}}^{3}=N$ is the density of the quasi-particles, in this case the holes. We may now apply the above formula for the susceptibility taking the second derivative with respect to $B_{0}$. When referring to the magnetic moment, we observe that the $B_{0}$ dependence in the second term in the brackets drops out. Hence, only the last term contributes, yielding

$\chi_{\mathrm{h}} \approx-\frac{1}{12} \frac{N}{T}\left(\frac{q_{\mathrm{h}} \hbar}{M_{\mathrm{h}}}\right)^{2}$

for the contribution of holes to the plasma susceptibility.

\section{Summary}

Plasmas are paramagnetic. This is a consequence of Pauli paramagnetism caused by the electron (and ion) spin. This paramagnetism is reduced by one-third by the orbital Landau diamagnetism of electrons (and ions). Above we have demonstrated that electron holes, once generated in plasma, provide another contribution to orbital diamagnetism, thus further reducing the bulk paramagnetism of the hole-carrying plasma.

One should note that the last above expression (like Landau's original form for electrons) is classical though it has been obtained via application of the quantum mechanical arrangement of hole energy levels. Even though the interior of the hole corresponds to positive charging, the susceptibility generated is negative and thus adds to the diamagnetism of the plasma in the region of the electron holes causing a local depression of the magnetic field here. This is clearly the effect of orbital motion of the hole, which, like for any charged particle, counteracts the external magnetic field force.

It is most interesting to note that the magnetic moments of the holes derived in our previous paper (Treumann and Baumjohann, 2012) do not contribute to susceptibility, a conclusion which had classically been expected from "van Leeuwen's theorem" (e.g. Huang, 1987; Kittel, 1963). On the 
contrary, they cause an amplification of the magnetic field inside the hole, which counteracts the global effect of the susceptibility. Thus from the point of view of the hole, magnetic moment carrying holes behave locally paramagnetic.

On the other hand, the susceptibility of the hole gas applies to the entire hole-carrying volume being proportional to hole density $N$ and the square of the hole-charge-to-mass ratio. The latter will not differ from ratio $e / m_{\mathrm{e}}$ by more than one order of magnitude taking into account that $q_{\mathrm{h}}$ results from the missing electrons in the hole, while $M_{\mathrm{h}}$ counts the masses of the electrons which are present in the hole (Treumann and Baumjohann, 2012).

The main quantity which determines the susceptibility is the hole density $N$. This is naturally a number substantially less than the plasma density. For this reason the effect of the susceptibility and the resulting diamagnetism will be small. The main magnetic effect of electron holes, as exemplified in our former paper, remains the amplification of the magnetic field along the flux tube embedding the hole. The hole behaves like a magnetically active medium by amplifying the field and, as a secondary effect, adding to diamagnetism via contribution to the magnetic Landau susceptibility of the plasma. This diamagnetism of holes is an additional though small contribution to the reduction of the electronic Pauli paramagnetism of the plasma.

Acknowledgements. This research is part of a Visiting Scientist Programme at ISSI, Bern, executed by R. A. Treumann in $2006 / 2007$. Hospitality of the ISSI staff is thankfully recognised.

Topical Editor L. Blomberg thanks one anonymous referee for her/his help in evaluating this paper.

\section{References}

Anderson, L., Ergun, R. E., Tao, J., Roux, A., LeContel, O., Angelopoulos, V., Bonnell, J., McFadden, J. P., Larson, D. E., Eriksson, S., Johansson, T., Cully, C. M., Newman, D. L., Goldman, M. V., Glassmeier, K.-H., and Baumjohann, W.: New features of electon phase space holes observed by the THEMIS mission, Phys. Rev. Lett. 102, 225004, doi:10.1103/PhysRevLett.102.225004, 2009.
Buneman, O.: Instability, turbulence, and conductivity in current-carrying plasma, Phys. Rev. Lett., 1, 8-9, doi:10.1103/PhysRevLett.1.8, 1958.

Buneman, O.: Dissipation of currents in ionized media, Phys. Rev., 115, 503-517, doi:10.1103/PhysRev.115.503, 1959

Davidson, R. C.: Methods in nonlinear plasma theory, Academic Press, New York, chapter 4, 1972.

Du, A., Wu, M., Lu, Q., Huang, C., and Wang, S.: Transverse instability and magnetic structures associated with electron phase space holes, Phys. Plasmas, 19, 159-162, doi:10.1063/1.3561796, 2011.

Huang, K.: Statistical mechanics, 2nd Edition, John Wiley \& Sons, New York, chapter 11, 1987.

Kittel, C.: Quantum theory of solids, John Wiley \& Sons, New York, chapter 17, 1963.

Landau, L. D.: Diamagnetismus der Metalle, Zeitschrift für Physik, 64, 629-637, 1930.

Landau, L. D. and Lifshitz, E. M.: Quantum Mechanics, Pergamon Press, New York, chapter 15, 1965.

Meiss, J. D. and Horton Jr., W.: Drift-wave turbulence from a soliton gas, Phys. Rev. Lett., 48, 1362-1364, doi:10.1103/PhysRevLett.48.1362, 1982.

Newman, D. L., Goldman, M. V., and Ergun, R. E.: Evidence for correlated double layers, bipolar structures, and very-lowfrequency saucer generation in the auroral ionosphere, Phys. Plasmas, 9, 2337-2343, doi:10.1063/1.1455004, 2001.

Tao, J. B., Ergun, R. E., Anderson, L., Bonnell, J. W. Roux, A., LeContel, O., Angelopoulos, V., McFadden, J. P., Larson, D. E., Cully, C. M., AQuster, H.-U., Glassmeier, K.-H., Baumjohann, W., Newman, D. L., and Goldman, M. V.: A model of electromagnetic electron physe-space holes and its application, J. Geophys. Res., 116, A11213, doi:10.1029/2010JA016054, 2011.

Treumann, R. A. and Baumjohann, W.: Advanced Space Plasma Physics, Imperial College Press, London, chapter 8, 1997.

Treumann, R. A. and Baumjohann, W.: Magnetic field amplification in electron phase-space holes and related effects, Ann. Geophys., 30, 711-724, doi:10.5194/angeo-30-711-2012, 2012. 